\title{
Anthropology of knowledge
}

\author{
Емм а Cон е м Max Planck Institute for Evolutionary Anthropology \\ and Max Planck Institute for Psycholinguistics
}

\begin{abstract}
Explanatory accounts of the emergence, spread, storage, persistence, and transformation of knowledge face numerous theoretical and methodological challenges. This paper argues that although anthropologists are uniquely positioned to address some of these challenges, joint engagement with relevant research in neighbouring disciplines holds considerable promise for advancement in the area. Researchers across the human and social sciences are increasingly recognizing the importance of conjointly operative and mutually contingent bodily, cognitive, neural, and social mechanisms informing the generation and communication of knowledge. Selected cognitive scientific work, in particular, is reviewed here and used to illustrate how anthropology may potentially richly contribute not only to descriptive and interpretive endeavours, but to the development and substantiation of explanatory accounts also.
\end{abstract}

In their various roles as perceivers, learners, recorders, communicators, and theorists of knowledge, anthropologists have long recognized the central importance of bodily experience in human knowledge. As learners of varied forms of cultural knowledge, they maintain and demonstrate the importance of 'being there' as experiential participants and observers. As communicators of knowledge, they are challenged to transcribe their experiences into forms of knowledge that are faithful to the richness of the data. And as fully engaged participants in the myriad aspects of human behaviour across variable cultural and learning contexts, anthropologists are uniquely positioned to generate precise descriptive and theoretical accounts of the making of diverse kinds of knowledge. The papers in this volume illustrate these aspects of 'embodied' anthropological inquiry, enhancing our appreciation not only of the diversity of learning environments with which anthropologists now engage, but also of the challenges that any explanatory account of knowledge-making faces.

Three challenges are especially clear. First, these papers conjointly demonstrate that to address satisfactorily the broad framing questions, concerning 'how we know' and 'how we come to know', we need more than a single explanatory account. As the contributors have so vividly shown, what we know takes many different forms. The social and cognitive mechanisms and processes by which different forms of knowledge 
are generated are multiple, involve different activating conditions, and produce different outcomes. Second, the generation of explanatory accounts of knowledge-making across these diverse forms necessarily requires the joint engagement of multiple disciplines and modes of inquiry. If we truly aspire to understand 'how we come to know', to espouse theories of knowledge acquisition, storage, retrieval, and communication processes, and to account for the importance of bodily and mental states in learning and performance, we simply cannot afford to ignore the vast and increasingly sophisticated scholarship on such issues in neighbouring disciplines. Third, we need precise empirical questions and testable hypotheses that are both generated from and generative of relevant data. The testing of precise hypotheses about the social and cognitive mechanisms underpinning and facilitating the transmission of knowledge may not immediately strike one as a particularly 'anthropological' challenge. Without clarity, precision, and methodological rigour, however, theoretical claims ultimately remain empirically intractable, unsubstantiated, and, therefore, of obscure value to the whole enterprise.

Many more general observations could be listed. The challenges are great. In this brief discussion, however, I will attempt to support these three observations, not simply as a synoptic take-home message, but as a series of guiding principles for future anthropological scholarship on human knowledge. Rather than abstract and address each of the above three points in turn, I will demonstrate their inter-reliance in practice, focusing on a central theme of this special issue, 'How do bodily factors influence the making of human knowledge?' The preceding papers offer rich descriptive analyses that point to the pervasiveness and centrality of 'embodied knowledge' in cultural transmission, and that characterize the social complexity of transmissive processes (see also Hutchins 1995; Lave 1988). But what of the mechanisms that establish and channel such knowledge? To echo Downey, what are the material dimensions of the learning process? How do these permit and constrain the transmission of culture? In the space available, I will attempt to offer some general statements, partial answers, and guiding principles relevant to these questions.

\section{Grounded cognition}

Cultural transmission - i.e. the emergence, acquisition, storage, and communication of ideas and practices - is powerfully influenced by the physical context in which it occurs. More specifically, what we know depends upon the brains, bodies, and environments in and among which transmission occurs. Disciplines differ with regard to the variable emphasis they place on neuro-cognitive, bodily, and social-historical factors in cultural transmission. Increasingly, however, researchers across the human and social sciences are recognizing that the bodily, cognitive, neural, and social mechanisms that permit and constrain knowledge transmission are conjointly operative and mutually contingent.

In cognitive scientific models, the traditional view that knowledge resides neurally independently of the mode-specific route by which it was acquired is gradually losing ground. In recent years, a novel framework has emerged, which presents cognitive processes such as perception, conception, attention, memory, and motivation, as 'grounded' in their physical context (Barsalou 2008). According to this view, knowledge resides in modality-specific neuro-cognitive systems (e.g. those that process vision, movement, audition, emotion, motivation, etc.) and is re-activated via the partial simulation of the cognitive and bodily states, social interactions, and environmental situations that contributed to its acquisition. For example, there is evidence to suggest 
that in order for pianists to identify whether a musical recording is of their own playing, they tacitly and internally simulate the motor actions that compose the performance (Repp \& Knoblich 2004). Importantly, these recent approaches do not conflate brains, bodies, and environments, or see all or any forms of knowledge as equivalently dependent on each, but rather they recognize specific and varied causal linkages among them. Specific body states, for example, have been shown to produce specific cognitive states (e.g. the activation of smiling musculature by clenching of a horizontally aligned pencil between one's teeth produces positive affect), and specific social stimuli produce specific cognitive states (e.g. perceiving another's laughter can produce positive affect in self) (see Barsalou, Niedenthal, Barbey \& Ruppert 2003).

How might this scholarship contribute to our understanding of the micromechanisms underpinning human knowledge? The ethnographic analyses presented here compellingly demonstrate the centrality of bodily states in the making of knowledge: for example, in how learning to listen, learning to weave and embroider, and learning to refine and attune one's sense to a novel skill domain (Makovicky, Portisch, Rice, Venkatesan). The complementary scholarship on grounded cognition, and investigations of implicit cognitive and behavioural phenomena, further indicate that cognitive, affective, and bodily states are intimately interconnected across an exceedingly broad and diverse range of knowledge forms. Research into the tacit linkages between concepts and bodily states demonstrates that embodied knowledge pervades even basiclevel conceptual categorization. In a well-known study by Bargh, Chen, and Burrows (1996), when social stereotypes associated with particular words were tacitly primed, embodied effects were produced. Adults primed with the word elderly, for example, took longer to walk from the laboratory to the elevator than adults in a control condition. The priming of the stereotype associated with elderly, particularly the assumption that the elderly tend to move slowly, appeared to generate corresponding bodily effects in the participants. Similar effects were obtained in another study by Aarts and Dijksterhuis (2002), in which participants were primed with the names of fast or slow animals (such as cheetah or turtle).

Barsalou explains such effects in terms of what he calls 'modal re-enactment'. According to this account, knowledge is not stored in some sort of neural filing cabinet, detached from the structures that are activated in its acquisition. Rather, the retrieval of knowledge entails the partial re-enactment of the very situation(s) that led to its encoding. Even retrieval of the most basic information pertaining to everyday objects and entities entails simulation of the situation of 'being there' with those objects and entities. Barsalou and colleagues, for example, asked research participants to produce properties for objects that would typically be found either above them (e.g. bird) or below them (e.g. worm). When producing properties for objects above them, participants were more likely to look up, and to lift their faces and hands upwards, than when thinking about objects typically found below them (see Barsalou et al. 2003).

These findings, and those of an expanding body of evidence, support the view that simulations underlie conceptual processing. In other words, the neural systems that produce experiences are activated in their subsequent representation - the modalityspecific states activated by an affective, visual, and motor experience, such as a bungee jump, are used in subsequent performances, descriptive commentaries, and so on, of that experience. This account can help to explain why the re-description of such kinds of experiences in propositional language fails adequately to communicate many of their most keenly felt dimensions, or why in the everyday telling of jokes and stories we often 
end up resigning to the conclusion that 'you had to be there' (or succumbing to the temptation to embellish).

Applying and developing theories, findings, and methodologies from this new framework of 'grounded cognition', anthropologists can strive to identify more precisely how body, brain, and environment inform and constrain the making of knowledge. What we mean by 'embodiment', as a term and perhaps even an 'approach' or 'paradigm' in anthropology, is all too frequently obscured in protracted chains of metaphorical reinterpretation and re-formulation. Of course, the examination and refinement of our analytical concepts are essential to establishing their interpretative utility. If we wish not only to describe and interpret, however, but also to explain how the body is implicated in knowledge acquisition, problematized concepts must eventually be supplemented with operationalized concepts. As Downey (this volume) argues, to account for how knowledge is acquired, we need to engage with the organic matter of the body, with specific material, physical, and neurological dimensions, and how these impact perceptual, conceptual, behavioural, and social phenomena, and vice versa. In his introduction, Marchand lists numerous scholars in the cognitive sciences, including anthropology, who have already done so. Considerable scope exists for sustained and more widespread involvement in such an enterprise.

Many anthropologists, of course, are uncomfortable with the idea of simply borrowing findings from cognitive sciences on the factors contributing to patterns of knowledge transmission. The relevant questions may not have been helpfully framed and investigated, and sample populations used are often unrepresentative along various important dimensions. Consequently, anthropologists are increasingly developing their own - or collaborative - scientific research programmes born out of their fieldwork observations (e.g. Astuti 2001; Astuti \& Harris 2008; Barrett \& Behne 2005). Recognizing that factors underlying patterns of transmission observed may not readily be discovered or confirmed through immersive participation and observation, direct interview, and other standard ethnographic techniques, these anthropologists have adapted and incorporated methods developed outside of anthropology that probe for out-of-awareness knowledge and reasoning biases. Appreciating more fully the range of factors - cognitive, social, environmental, etc. - that govern behaviour, anthropologists can develop plausible accounts for patterns of behaviour that may otherwise remain unsolvable puzzles. Allow me to illustrate with an example from my own work.

\section{The cognition of possession}

Following fieldwork with a group of Afro-Brazilian spirit mediums in Belém, northern Brazil, numerous phenomena puzzled me as I reflected upon the behaviours and statements of my research participants. One such puzzle concerned the apparent inconsistencies in the ways in which my friends in the field talked about and behaved around possessed individuals. Possession (incorporação), I was frequently told, involves the entry of a spirit into a person's body, specifically the person's head. When the spirit comes into the person's body, the person leaves, and where the person is thought to go depends upon whom one asks. Some, for example, suggested that they lie down and sleep; others said that they fly away; others said that they remain in the spot they were at the moment the spirit entered their body. In contrast, there is a broad consensus on what possession entails for the body and for the spirit. The spirit inhabits the body temporarily, taking control of it and using it in the service of mediumistic activities, 
such as healing and counselling. Subsequent behaviours are said to be attributable to the intentions, desires, and so on, of the spirit, not of the medium.

These descriptions of possession, however, often conflicted with observers' commentaries about particular behaviours in particular possession episodes. Mediums were often teased for dancing or singing inadequately or for behaving inappropriately while possessed. People's interactions with mediums when possessed displayed striking continuities with their interactions with mediums when puro, or not possessed. For example, if a particular person harboured negative feelings toward a particular medium, these attitudes appeared to influence behaviours toward that medium also when possessed.

The community leader (pai-de-santo) once related a story in which a number of core members (filhos-de-santo) committed severe infractions of the house rules while possessed. He described how four filhos, possessed with spirit entities, helped themselves to alcoholic drinks that belonged to the house. The infraction was two-fold: consumption of alcohol was strictly forbidden in the house, and the alcohol consumed on this occasion was specifically and exclusively reserved for ritual libations to the spirit entities of the house. The pai-de-santo's telling of the story revealed a certain level of ambivalence about whom to blame - the filhos, or the spirit entities possessing them.

He began by naming names, not of spirit entities but of filhos. Then, he itemized his reactions to the situation:

First, I think that it wasn't necessary for the entities to do this. 'Look, I want to drink - ah, it's not ok - alright then, I'll go away'. Fine! Second, the entities know the regime of the house. Third, they were stealing. Sure, this family [of spirits] is said to be fond of their drink, but ... I couldn't get over it and I'm still not over it.

Moments later, the blame seemed to be reside more in the direction of the filhos:

Look, the most important people of the house - in terms of hierarchy - were involved. The most interesting thing is that the spirit entities know that these people cannot drink ... I just don't understand this at all.

And by the end of his record of the incident, it was quite clear that his disappointment was chiefly with the filhos:

I thought that I was composing a stable, rigid, obedient society ... I thought that I could die in peace - that the community would continue on, but now I do not have this assurance. If I die, what is going to happen? ... I thought that I had a group of filhos-de-santo who were faithful, and sincere, but unfortunately this was not the truth. A betrayal within a house means a lot to me, especially since I have open communication [lit. 'an open game'] with the filhos-de-santo.

An obvious question, then, concerns the ways in which various forms of knowledge, at various levels of awareness, interact and inform one another in reasoning about possession. Possession, in the abstract, entailed that the filhos were no longer present as social agents, having been displaced from their bodies by incorporeal spirit entities. In actual possession episodes, and real-time interpersonal interactions, however, it appears that this abstract definition of possession did not consistently inform people's representations of agency, intentionality, and responsibility. Other anthropologists have reported similar patterns, but few have attempted to explain precisely how these 
patterns arise. Niko Besnier, for example, states that 'spirits and their world cannot be understood through a search for a resolution of such ambiguities and contradictions; rather, these qualities must be perceived as constitutive of the very nature of spirits' (1996: 76, original emphasis).

I suggest that a range of cognitive scientific findings can facilitate the development of an explanatory understanding of apparent contradictions in what people say, and in what people do, not only in possession scenarios, but across variable social, cultural, environmental, motivational, and emotional contexts. Explanations of such phenomena are often hastily dismissed for their purported imposition of coherence and rigidity on 'fluid', 'shifting', 'complex', and 'conflictual' cultural processes and discourses. Yet a considerable and growing literature investigating how human psychology reacts with bodily, social, and environmental stimuli now points to the presence of significant constraints and predictable biases on human reasoning.

The perception and interpretation of possession scenarios are guided by a set of implicit mental tools that deal with social perception in a broad range of interpersonal contexts. Through further ethnographic and experimental research on the cognition of possession, psychologist Justin Barrett and I discovered that the abstract definition of possession - that entailing a displacement of the medium's agency upon the entry of the spirit - does indeed appear to be underpinned by strong cognitive biases. As a result, the structure of this concept appears to be significantly more memorable, for example, than comparable possession concepts (e.g. that entail the merging of host and spirit agency in the host's body). This may explain, in part, widespread incidence cross-culturally of such displacement concepts (see Cohen \& Barrett 2008a; 2008b).

In real-time perception of possessed individuals, however, a different set of cognitive mechanisms is activated, biasing individuals toward alternative representations of possessed individuals. Neuroscientific and psychological evidence on the processes underpinning the perception of faces, for example, indicates that the observation of the face of a known individual activates affective and semantic information that the observer holds regarding that individual (Leveroni et al. 2000; Shah et al. 2001). Because the pathway of activation - from systems involved in face perception, to those involved in face recognition, and ultimately person recognition - is automatic, we cannot readily attribute new identities to familiar faces. In our observations of how people represent possession episodes, we should therefore expect ambivalence and ambiguity in what observers say and do.

Drawing from our understanding of the implicit and explicit cognitive processes involved in person recognition, novel predictions can be generated concerning the variable importance of different bodily and behavioural cues for processes of person perception in possession and related contexts (e.g. actor type-casting). What bodily and behavioural transformations are likely to enable people more readily and consistently to represent a person as 'no longer present' and their body as now inhabited by a different identity? We might predict, for example, that the wearing of facial masks would produce interestingly different effects from situations in which such accoutrements are not used. What kinds of motivational and emotional factors, and other interpersonal expectations, are important for explaining the ambivalence with which the pai-de-santo understood the behaviour of the possessed filhos described above?

These questions are possibly interesting, but they are certainly tough to answer. How people reason about possession is produced by the interaction of brain, body, and social environment, and varies according to the ways in which elements of these three 
domains are differently configured across different contexts. This is not tantamount to an incoherent and impenetrable chaos, however. The material properties of each of these dimensions have variable, predictable, and often measurable effects on the making, storage, retrieval, and communication of knowledge. Anthropologists cannot always depend upon other disciplines to investigate how these dimensions contribute to the patterns of behaviour and cultural transmission they observe. Rather, generating plausible accounts of the complexities of knowledge-creation and -activation often requires a concerted, collaborative interdisciplinary effort, and the sharing of methodological tools, data, and theoretical insights.

\section{From fieldwork to fMRI?}

Talk of the centrality of bodies and brains in knowledge transmission, together with appeals to neuro-cognitive theories on simulated re-enactments, and neuroscientific evidence on person recognition processes, may give the impression that the explanatory approach I am advocating necessarily requires that, at a certain point, we throw away our notepads and pencils for expensive brain-scanning gadgetry and white coats. The choice, however, is not between social, cultural, and historical phenomena, on the one hand, and brain mechanisms, on the other; nor do traditional fieldwork methods and flashy scanning methods even remotely constitute the complete battery of potentially relevant techniques available. Conventional methods in the psychology of learning and knowledge, including systematic observational techniques and eye-tracking, reactiontime, implicit priming, and recall studies, are highly relevant to perennial anthropological concerns and questions about the transmission of culture.

Take imitation, for example. Imitative learning has been a central focus of research in developmental psychology. Despite the richness and importance of findings in this area, the neuroscientific discovery that imitative capacities are neurally grounded in a specially dedicated class of brain cells, called mirror neurons, has fast become one of cognitive science's most successful exports to the social sciences. Mirror neurons have received special attention, in particular, in discussions of skill acquisition and cultural transmission more broadly (including in this collection). That they operate unconsciously and automatically is often taken to suggest that learned motor behaviours are simple emulations, or behavioural copies, of observed behaviours in others. Much of cultural transmission is, by extension, explained in terms of what is, in effect, a highfidelity neural copy machine. Research with infants, however, suggests that imitation does not consist only of re-enactments of demonstrated action. Rather, imitative behaviours are frequently selective, and the inferential processing biases that govern selection are firmly in place early in infancy.

In a series of simple but groundbreaking studies György Gergely, Gergely Csibra, and colleagues first demonstrated not only that 12-month-old infants are able to attribute goals to observed actions, but that they can also assess the efficiency with which the goal is achieved via the specific act, and according to the physical constraints upon the agent acting (Gergely, Nádasdy, Csibra \& Bíró 1995; Csibra, Gergely, Bíró, Koós \& Brockbank 1999). They then investigated whether considerations of efficiency would influence 14-month-old infants' imitative behaviours. In an adaptation of a seminal study by Andrew Meltzoff, in which actions demonstrated by an adult actor were re-enacted by infants after a one-week period, Gergely, Bekkering, and Király (2002) showed that 14-month-olds tend to re-enact a goal-directed action only if they perceive the action to be an effective means of achieving the intended goal. Infants 
watched an actor switch on a light box using her forehead. In one condition, the actor's hands were free while she executed the action, and in the other condition, her hands were occupied (pretending to be cold, she had wrapped a blanket around herself, which she held with both hands). Experimenters report a significant difference in the number of infants who copied the demonstrated action between the two conditions. When the demonstrator's hands were free, 69 per cent of infants re-enacted the head action. When the demonstrator's hands were occupied, only 21 per cent of the infants copied the head action, with the remainder choosing instead to turn on the light with their hands. The experimenters conclude that 'the early imitation of goal-directed actions is a selective, inferential process that involves evaluation of the rationality of the means in relation to the constraints of the situation' (Gergely et al. 2002: 755).

Developmental studies such as these suggest that emulation - or simple behavioural copying - is a component capacity in cultural learning, but that it is recruited alongside additional cognitive capacities that enable us to detect goals and assess the relative efficiency and relevance of variable routes to achieving those goals (all of which may be facilitated through the activation of mirror-neuron circuits, see Barsalou 2008: 623; Gallese, Keysers \& Rizzolatti 2004). Where the functional rationale for a demonstrated behaviour is unknown, however, actions are more likely simply to be emulated. This is because, to the naïve observer, the relevant elements of the action may not be readily distinguishable from the irrelevant components. Gergely and Csibra (2006) suggest that selective transmission of relevant knowledge is enhanced by demonstrators, or teachers, through a variety of what they call 'ostensive-communicative cues'. Such cues include eye contact, contingent reactivity, gaze shifting, pointing, behavioural demonstration, eyebrow flashing, and so on. Gergeley and Csibra propose a bilateral humanspecific pedagogical inclination to 'teach' each other, or to transmit relevant information via the use of these ostensive cues, and to learn from each other, or to be receptive to such cues (Gergely, Egyed \& Király 2007). Such a 'pedagogical stance', if borne out, would be an extremely important component of any account of cultural transmission, and, indeed, of human sociality at large. Ethnographic evidence on whether ostensive-communicative cues are as widespread as this view suggests will surely be required to test these hypotheses. In those cultural contexts and learning situations where ethnographers have reported the absence of direct propositional teaching, do we none the less encounter a range of ostensive-communicative cues in transmission? Is the repertoire of such 'pedagogical tools' broadly similar, and are there recurrences in the patterns of activation of various components of the repertoire?

These tools and findings can demonstrate the relevance and importance of methodological pluralism beyond fieldwork and brain scans. This issue is more than methodological, however. By leaping from culture to brains and back, and bypassing the cognition in between, we risk missing what is happening at the psychological and behavioural levels. Explaining phenomena at the socio-cultural level in terms of the patterns of activation of neurons in individual brains is analogous to explaining a computer hardware failure in terms of the atomic structure of the materials of which it is composed. It is at the level of the psychological mechanisms, processes, regularities, and biases that constitute (and that may be constituted by) the individual and distributed ideas, expectations, intentions, behaviours, beliefs, feelings, attitudes, and so on, that make up a socio-cultural environment that we will potentially discover factors that meaningfully account for macro-cultural patterns. If we are to concentrate our collaborative efforts, then, in any domain of the broad aggregate of disciplines and 
specialisms that is cognitive science, an alliance with psychology is likely to be a particularly fruitful one.

Because of the complexity of human culture, sociality, behaviour, and thought, questions about the factors contributing to patterns of knowledge transmission are rarely settled with any single set of tools or findings. How we come to know necessarily entails complex and contingent interactions among brains, bodies, and environments. There is an important role for anthropologists in identifying these interactions - not only as sensitive interpreters of human behaviour, but as methodical describers of human behaviour as it may be witnessed through a potentially broad variety of techniques. The contributions to this volume offer both rich data and probing questions for accounts of bodily knowledge, moving anthropology toward a position from which the discipline can further its collaborative engagement with relevant theories and findings in the cognitive sciences on cognition, embodiment, learning, and knowledge. As I have attempted to argue, the generation of insightful interpretative analyses of behaviour need not be the end-point of the ethnographic process. Faithful descriptions and analyses can engender data-driven questions and hypotheses about the causal mechanisms and processes that enable us to 'come to know'. Ultimately, however, 'being there' and the importance of sustained participant-observation in the generation of descriptive portrayals of human behaviour is powerfully upheld by emerging 'grounded cognition' models of human knowledge. A rich understanding of local concepts and categories as they inform ideas and practices, and the progressive departure from exclusively ethnocentric (mis) understandings, is best achieved via direct and repeated exposure to the linguistic, social, bodily, motivational, and affective contexts in which these concepts and categories appear. The papers in this volume are surely strong testimony to this fact.

\section{REFERENCES}

Aarts, H. \& A. Dijksterhuis 2002. Category activation effects in judgement and behaviour: the moderating role of perceived comparability. British Journal of Social Psychology 41, 123-38.

Astuti, R. 2001. Are we all natural dualists? A cognitive developmental approach. Journal of the Royal Anthropological Institute (N.S.) 7, 429-47.

\& P. HARris 2008. Understanding mortality and the life of the ancestors in rural Madagascar. Cognitive Science 32, 713-40.

Bargh J.A., M. Chen \& L. Burrows 1996. Automaticity of social behaviour: direct effects of trait construct and stereotype activation on action. Journal of Personality and Social Psychology 71, 230-44.

BArRett, H.C. \& T. Behne 2005. Children's understanding of death as the cessation of agency. Cognition 96, 93-108.

Barsalou, L.W. 2008. Grounded cognition. Annual Review of Psychology 59, 617-45.

- P.M. Niedenthal, A.K. Barbey \& J.A. Ruppert 2003. Social embodiment. In The psychology of learning and motivation, vol. 43 (ed.) B.H. Ross, 43-92. San Diego: Academic Press.

BesNier, N. 1996. Heteroglossic discourses on Nukulaelae spirits. In Spirits in culture, history and mind (eds) J.M. Mageo \& A. Howard, 75-97. New York: Routledge.

Cohen, E. \& J. BARRETT 2008a. When minds migrate: conceptualizing spirit possession. Journal of Cognition and Culture 8, 23-48.

$-2008 b$. Conceptualizing spirit possession: ethnographic and experimental evidence. Ethos $36,245-66$.

Csibra, G., G. Gergely, S. Bíró, O. Koós \& M. Brockbank 1999. Goal attribution without agency cues: the perception of 'pure reason' in infancy. Cognition 72, 237-67.

Gallese, V., C. Keysers \& G. Rizzolatti 2004. A unifying view of the basis of social cognition. Trends in Cognitive Science 8, 396-403.

Gergely, G., H. BekKering \& I. Király 2002. Rational imitation in preverbal infants. Nature 415, 755. 


\section{S202 EMMA COHEN}

\& G. Csibra. 2006. Sylvia's recipe: the role of imitation and pedagogy in the transmission of human culture. In Roots of human sociality: culture, cognition, and human interaction (eds) N.J. Enfield \& S.C. Levinson, 229-55. Oxford: Berg.

, K. Egyed \& I. Király 2007. On pedagogy. Developmental Science 10, 139-46.

Z. NÁdAsdy, G. Csibra \& S. Bíró 1995. Taking the intentional stance at 12 months of age. Cognition 56, 165-93.

Hutchins, E. 1995. Cognition in the wild. Cambridge, Mass.: MIT Press.

Lave, J. 1988. Cognition in practice. Cambridge: University Press.

Leveroni, C.L., M. Seidenberg, A.R. Mayer, L.A. Mead, J.R. Binder \& S.M. Rao 2000. Neural systems underlying the recognition of familiar and newly learned faces. Journal of Neuroscience 20, 878-86.

Repp, B.H. \& G. KNOBLICH 2004. Perceiving action identity: how pianists recognize their own performances. Psychological Science 15, 604-9.

Shah, N., C. Marshall, O. Zafiris, A. Schwab, K. Zilles, H.J. Markowitsch \& G.R. Fink 2001. The neural correlates of person familiarity: a functional magnetic resonance imaging study with clinical implications. Brain 124, 804-15.

\section{Anthropologie de la connaissance}

\section{Résumé}

Les comptes-rendus portant sur l'émergence, la diffusion, la conservation, la persistance et la transformation des connaissances se heurtent à de nombreuses difficultés théoriques et méthodologiques. Bien que les anthropologues soient particulièrement bien placés pour affronter ces défis, des progrès considérables pourraient être réalisés en la matière dans le cadre d'une approche conjointe avec des disciplines voisines menant des recherches connexes. Les adeptes du décloisonnement des sciences humaines et sociales reconnaissent de plus en plus l'importance des interactions et interdépendances entre mécanismes physiques, cognitifs, neurologiques et sociaux dans la production et la communication des connaissances. Des travaux scientifiques choisis, en matière de cognition en particulier, sont examinés et utilisés pour illustrer la manière dont l'anthropologie pourrait apporter une riche contribution non seulement aux tâches descriptives et interprétatives, mais aussi à l'élaboration et la mise à l'épreuve de comptes-rendus explicatifs.

Emma Cohen is a researcher in the Research Group for the Comparative Cognitive Anthropology attached to the Max Planck Institute for Evolutionary Anthropology, Leipzig, and the Max Planck Institute for Psycholinguistics, Nijmegen. She has conducted fieldwork on an Afro-Brazilian religious tradition in Belém, northern Brazil, focusing primarily on concepts, behaviours, and practices associated with spirit possession. Her publications include The mind possessed (Oxford University Press, 2007). She is currently researching the ways people (across cultural and religious contexts) represent the relationship between minds, bodies, and persons.

Research Group for Comparative Cognitive Anthropology, Max Planck Institute for Evolutionary Anthropology, Deutscher Platz 6, 04103 Leipzig, Germany.emma_cohen@eva.mpg.de 